\title{
Proposed A New Multidimensional Face Recognition For Surgically Altered Faces In Security Surveillance
}

\author{
Dimple Chawla, Munesh Chandra Trivedi
}

\begin{abstract}
From previously carried out review results, authors have proposed a new multidimensional face recognition approach and applied on the sample database of sensitive medical images. The basic idea is to differentiate an individual's identity with its own core features extracted from local or global plastic surgery. Features are being highlighted to match pre-topost or post-to-pre surgery face images. The procedure may affect different face recognition algorithms working on linear or nonliner variations approach and that's what multidimensional approach is meant. Hence it proves that the performance of verified rank accuracy ratio has increased in face recognition for security surveillance.
\end{abstract}

Index terms: Face Recognition, Feature Extractors Algorithm, Principal Component Analysis (PCA), Independent Component Analysis (ICA), Local Binary Pattern (LBP), Fisher Discriminate Analysis (FDA), Local Feature Analysis (LFA)

\section{INTRODUCTION}

Face recognition technique is widely used for many data analytic approach in computer vision, data mining, and other bioinformatics for security surveillance etc. [1]. It follows the spatial geometry feature for identifying the distinguishing facial features in a face image. The application is defined for computer vision that uses to identify or to validate the person's identity [2]. An important difference with other biometric based system is the faces captured from some space through surveillance cameras are the distance that varies in input. Therefore, face recognition can be applied without knowing the subject that the face is being observed and can be altered. This makes face recognition suitable for finding fraud people, missing children or tracking down fugitive criminals using surveillance cameras [3].

Classification of Plastic surgery can define in two distinctive categories: local $\mathrm{n}$ global surgery [2]. Local plastic surgery is used for correcting defects, anomalies, or improving skin texture. For example- disease correcting local plastic surgery would be correcting jaw, teeth and nose structure, chin, forehead, and eyelids etc. Apart from local surgery, plastic surgery can be done for global change in the facial structure which is known as complete-face or full face-lift will be considered as global plastic surgery [3].This

Revised Manuscript Received on August 14, 2019.

Dimple Chawla, Research Scholar, PAHER University, Udaipur (Rajasthan), INDIA(email Id: chawladel@gmail.com)

Dr. Munesh Chandra, Trivedi, Dean (Academics), Rajkiya Engineering College, Azamgarh (Uttar Pradesh), INDIA(email Id: munesh.trivedi@gmail.com) type of medical procedure is suggested for cases where patients fall with fatal burn or trauma. Better name renowned as Digital Beautification, which completely changes the appearance of a person using laser resurfacing, photodynamic therapy or photo-rejuvenation treatments [4].

In this study, authors have followed to work upon surgically altered faces by studying the various plastic surgery procedures performed on different portion of the face such as forehead, nose, cheekbones', lip, chin etc. The study has proposed a new multidimensional approach for altered faces by implementing it and opposing its result with the traditionally proven results. The plastic surgery process may affect the texture, appearance, size and shape of facial regions after application on pre-surgical images. Therefore, it is difficult for face recognition algorithms to match a postsurgery with its pre-surgery face image.

\section{RELATED STUDY}

This paper, authors have continued their work from the outcome of previous paper [5], in which they have briefly reviewed the studies undertaken by others in past. There are numerous methods available for using the same algorithm that perform the post to pre comparison of the surgically altered faces \& authors have gone one step ahead by proposing a new multidimensional approach through design \& implementation, which helps in increase the performance accuracy ratio of the process. While applying the same face recognition algorithm system may affected the minor change induced in plastic surgery globally. It is also considered as holistic appearance features that capture the overall face appearance. Among the most popular worldly used methods are used their results here for comparative evaluation [6].

For the most popular analysis for face detection is Principal Component Analysis. It is an orthogonal linear transformation that converts the data to a new coordinate system mathematically. The maximum variance of the data lie on the first coordinate called as first principal component and the second coordinate as the second greatest variance, and so on [7]. The clear objective is to rotate rigidly on the coordinate axes as shown in Figure 1 of the p-dimensional linear space to new 'natural' positions (principal axes) so that coordinate axes correspond to the highest variance in data that principal axis 1 , axis 2 has the next highest

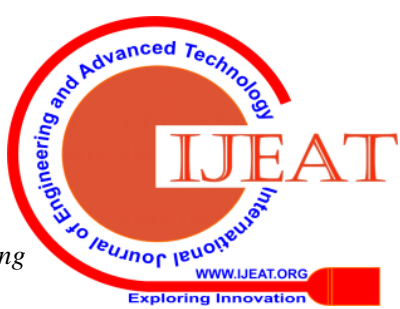


variance, and axis $\mathrm{p}$ has the lowest variance. The covariance among each pair of principal axes is zero, i.e. they are uncorrelated [8].

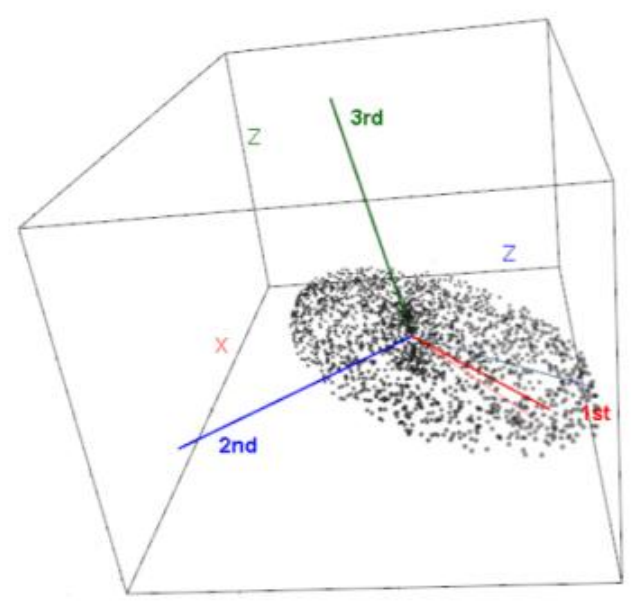

Figure 1: 3D projected plan

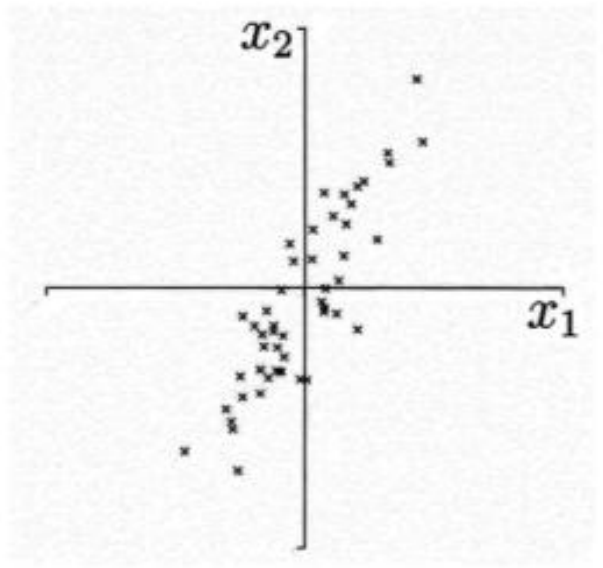

Figure 2: 2D Vector Space $(x 1, x 2)$

PCA is a standard technique for analyzing then visualizing the high dimensional data for data preprocessing, which helps in reducing the dimensionality (the number of variables). The key property of using Principal Component Analysis is optimal reconstruction, minimizing the squared reconstruction error and maximizing the variance of projected input vectors [9].

Linear Binary Pattern is an efficient nonparametric descriptor that uses great number of variation and is used to design the local structures of images into a feature vector mentioned in Figure 2. The implementation of LBP-based facial image analysis conducts the high range of application, which offers the better performance as well as to improve the robustness in one or more aspects are also highlighted [9]. The feature vector defines the purpose of mean to respond at different forms and orientations. Likewise, the LBP descriptor is able to extract the local feature information of face images, edges, peaks, and others. The LBP features are invariant to changes in scale and lighting effect. LBP has been recognised for simple and efficient method for invariant to monotonic greyscale transformation and illumination changes [10].
FARO (Face Recognition against Occlusions and Expression Variations) [11], is an image fractal based technique used for Partitioned Iterated Function System (PIFS), and FACE (Face Analysis for Commercial Entities) [11], which exploit in measuring the local features from the correlation index. Both methods use a localized regionbased like approach. The FARO performs recognition by dividing face into regions for significant meaning (eyes, nose and mouth) [11]. The second method, it performs the image into different extracted blocks of equal domain FAST Algorithm [12].

\section{PROPOSED ALGORITHM}

The Proposed algorithm described in Figure 3, works in 2 sections, one for pre surgical face image which follows in 3 steps as follows:

\section{A. Pre-Processing of Surgical face image:}

Firstly perform Face Detection Algorithm (PCA) on pre surgical face image, which is most expensive method to extract feature vectors and maximizes the use of variances from the extracted features.

\section{B. Feature Extraction Methods:}

It is the method to extract features with the help of extract Features(I, points) and returns its feature vectors, also known as descriptors in correspond to its locations and value. Also detect the corners using FAST algorithm and return corner Points of the face image. It is achieved to extract to encode the discriminative information required for face recognition for feature vector using techniques.

C. It is the method to extract features with the help of extract

Features(I, points) and returns its feature vectors, also known as descriptors in correspond to its locations and value. Also detect the corners using FAST algorithm and return corner Points of the face image. It is achieved to extract to encode the discriminative information required for face recognition for feature vector using techniques.

\section{Face Recognition Process:}

Under this process, a model is processed with face appearance and to classify them for matching the classifier model of pre surgical image to match with post surgical face image.



Figure 3: Proposed Algorithm Flowchart 
Second section for post surgical image works in following steps

i. Firstly generate the histogram of oriented gradients

ii. Download the image so to enhance the facial image on the focused area by using crop or resizing it called as normalizing it to the key interest area.

iii. Follows the Step 2 and 3 of pre surgical face image, where training and recognition process takes place.

iv. Lastly, matches both the classifier model, if it matches well then check the rank accuracy ratio for verification.

\section{SIMULATED WORK}

The proposed approach is implemented by MATLAB version R2017a, computer with Intel(R) Core(TM), i5$5200 \mathrm{CPU} @ 2.20 \mathrm{GHz}$ and windows 10. The system works in 2 phases, one for pre and another for post surgical face images respectively. During step1 and 2, authors have tried to eliminate the challenging problems like illumination, pose, aging factors etc. The whole proposed algorithm is tested on the self-owned database of sensitive medical facial images taken from hospital (Plastic surgery department) of pre and post surgical face images. The study has taken 200 sizes of medical scan database but here the result of only few of them has been taken in Figure 4.



Figure 4: Sample Database of pre and post surgical face images

\section{EXPERIMENTAL RESULT}

The entire experiment would have conducted on publicly available databases as well for frontal-face recognition, and then it would be considered as repetitive work like many other have already done. So, the whole experiments is done from the source of person present, and especially taken a consent which would be available on request. Surgical images involving pre surgical face images and post surgical face images served to demonstrate the efficiency of the proposed approach. We have randomly taken the database into pre-processing set and testing set, and then resize the image and normalize the pixel values to proof the result for successful match or not to vary result in $[0,1]$.

Firstly, both of the face images were passed to the face detector viz. Viola and Jones face detector to detect and then crop the area of interest. The detected face area (face, eyes, nose, mouth etc) marked using bounding box property of Images. Likewise, Figure 4(1a) bounding box of pre-image $[403,360,500,122]$ with image of $960 \times 1280 \times 3$ uint 8 and Figure 4(1b) bounding box of post-image [459,390,457,112]-960x1280x3 uint8 is shown as result in Figure 5 and Figure 6 respectively.

With the help of Viola and Jones Face detector techniques, Cascade Object Detector function purposely used to obtained object shown in Figure 7 and Figure 8 for pre-surgical image and post-surgical image. Weber's law normalization is used to neutralize the illumination variations in the images of the detected faces. Likewise, normalized eye region from Figure 7, 8 used to find out the Histogram of pre and post-image shown in figure 9 and Figure 10 defines performance on detection pre-to-post as well as on post-to-pre surgical face images.



Figure 5: Face Detection with Feature Extraction (Pre-Surgical Face)



Figure 6: Face Detection with Feature Extraction (Post-Surgical Face)

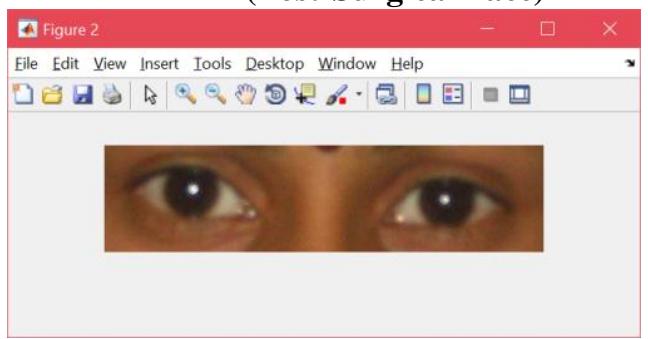

Figure 7: Normalize for Eye Detection (Pre-Surgical Face Image) 


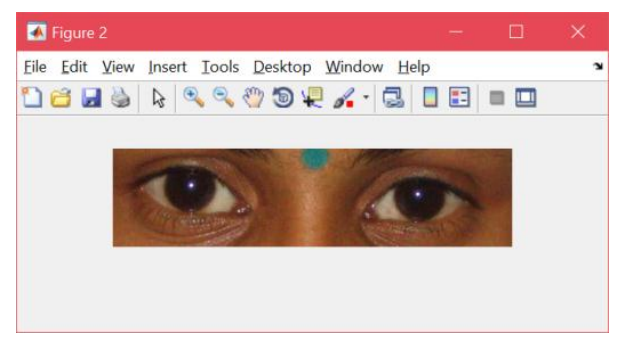

Figure 8: Normalize for Eye Detection (Post-Surgical

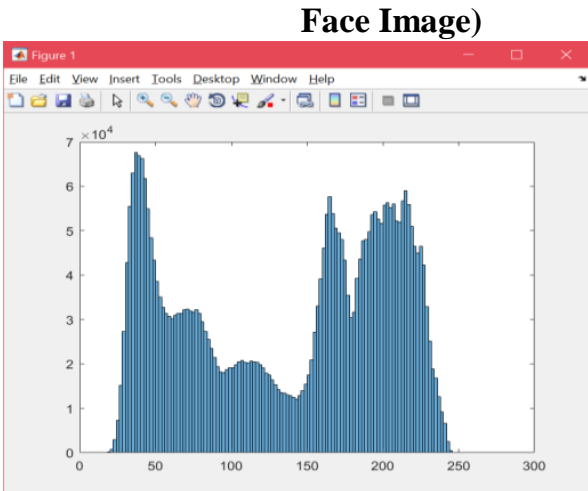

Figure 9: Histogram (Pre-Surgical Face Image)

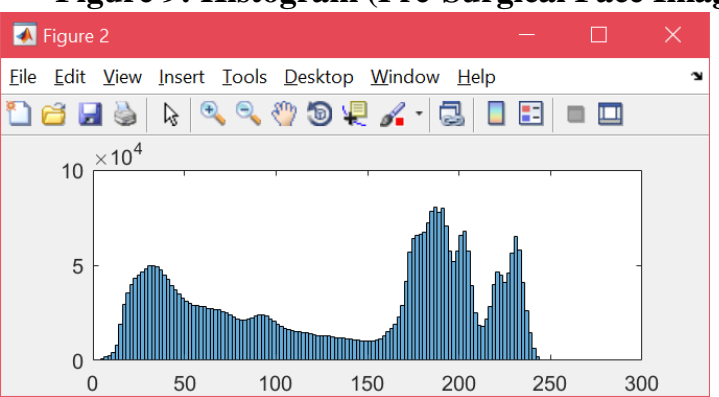

Figure 10: Histogram (Pre-Surgical Face Image)

While performing pre-processing, training and recognition, here the experiment and the comparison signify the traditional surgical face images on both its pre as well as post surgical image on skin-color model approach to determine facial appearance and variable lighting and background conditions shown in Figure 11. It have been noticed in an account on the face varying expressions and poses, the system will unable to calculate accuracy on detecting with noise and occluded face. That's why authors have chosen surgical images on keeping this factor aside.

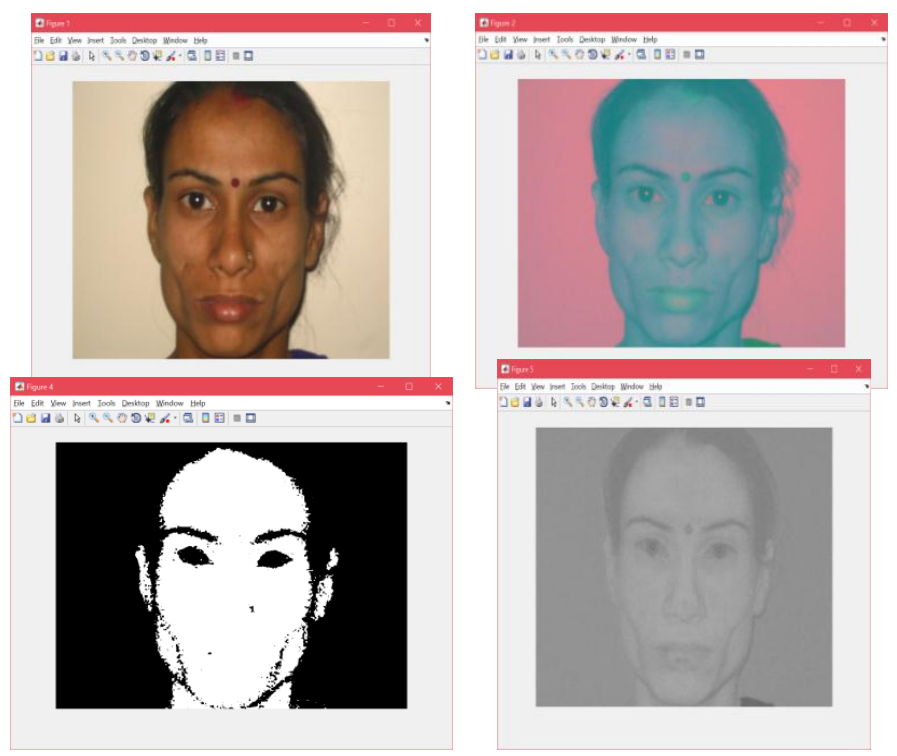

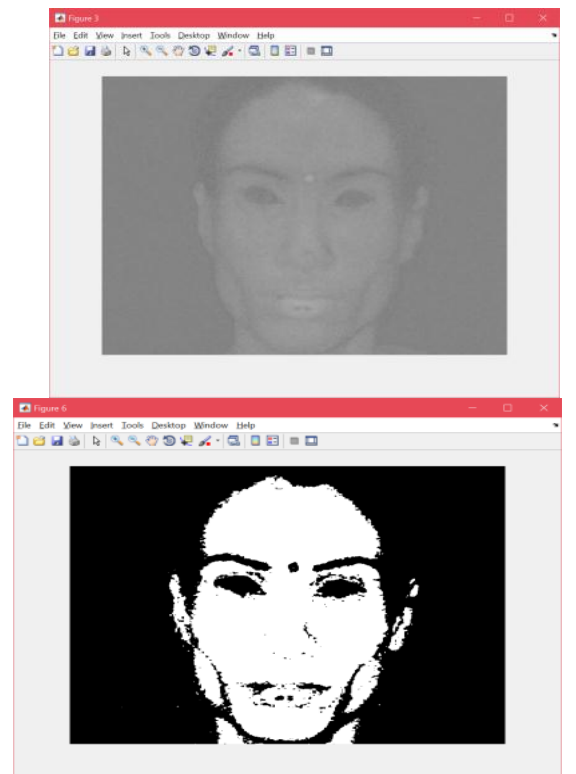

Figure 11: Skin-Color Model Applied On Pre-Surgical Image

After feature extraction and performance measure, the classifier model is created to match both surgical images. On the basis of PCA, LBP, FARO, and FACE, applied and used to match face descriptor which defines the composition of micro-patterns. The system using LBP as face descriptor has proven the classifier model is an appropriate decision. The conventional method for computing LBP in equation (1) from [13] at location $(x, y)$ for the input image is calculated as follows:

$$
\operatorname{LBP}(\mathrm{x}, \mathrm{y}) \quad=\operatorname{LBP}(\mathrm{x}, \mathrm{y})=\sum_{b=0}^{N-1} \mathrm{~S}(\mathrm{~Pb}-\mathrm{Pc}) 2 \mathrm{~b}
$$

where $\mathrm{Pb}$ is the estimated pixel value from neighbouring pixels, $P c$ is pixel value at $(x, y)$ and $S(z)$ defines the value 1 or 0 w.r.t $\mathrm{z}$ is greater than and equal to or less than zero.

There are two error metric techniques available for calculating i.e. Mean Square Error and Peak Signal to Noise Ratio. The collective squared error for pre surgical and post surgical face image is a measure of the peak error [14].

The mathematical formulae for

$$
\frac{1}{M \mathbb{N}} \sum_{\mathrm{y}=1}^{\mathrm{M}} \sum_{\mathrm{x}=1}^{\mathrm{N}}\left[\mathrm{I}(\mathrm{x}, \mathrm{y})-\mathrm{I}^{\prime}(\mathrm{x}, \mathrm{y})\right]^{2}
$$

(MSE) =

Using MSE, squared Error of LBP Histogram is generated in Figure 12. Lastly, the matching histogram relates to the two sets of facial images are fused together using the weighted sum rule. The performance is calculated for both the set of pre-to-post surgical image independently with the post-to-pre surgical image, and their performance being compared with the other plastic surgery process studied on the basis of the performance rank accuracy ratio of different altered facial images. 


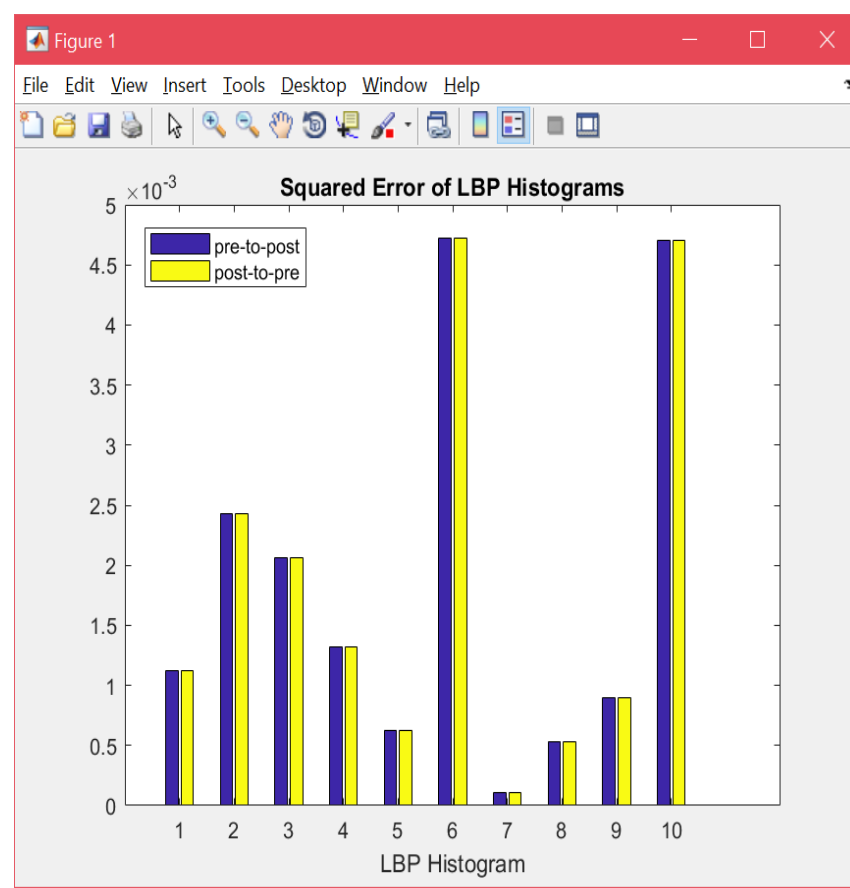

Figure 12: LBP Histogram Match

\section{CONCLUSION}

More than a very few face recognition methods concentrate on facial features when plastic surgically images being taken care off. Depending upon the detection method chosen, it would be possible to locate a face and its facial features in a pre-surgical to post-surgical face image with great accuracy. We have repeated each experiment for at least 10 times so that accuracy is calculated and also examined the performance on out of the bound conditions on MATLAB when multiple programs being called for the evaluation to identify the accurate match in smallest running time duration.

Table 1: Face Components Analysis

\begin{tabular}{|c|c|c|c|c|c|}
\hline & Post-Fa & e Com & onents & & \\
\hline & & Face & Nose & Mouth & Eyes \\
\hline \pm & Face & 1.00 & 1.00 & 1.10 & 1.00 \\
\hline & Nose & 1.00 & 1.00 & 1.00 & 1.00 \\
\hline 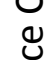 & Mouth & 1.17 & 1.00 & 1.00 & 0.90 \\
\hline $\bar{\alpha}$ & Eyes & 1.00 & 1.00 & 1.00 & 1.00 \\
\hline
\end{tabular}

However, the proposed system has achieved maximum verification accuracy with face component full match which is considered as the highest verification accuracy ratio reported so far. Which is also compared with the other stateof-the-art face recognition methods on plastic surgery studied in research and concluded the result in Table 1. While addressing the detecting and locating the facial features where accurate detection coverage has been observed like eyes, nose and face itself but the mouth within that face, more detailed analysis of the image is required because additional detection is coming across with the result that authors should keep an eye on it for future processing

\section{REFERENCES}

1. De Marsico M., Nappi M., Riccio D., Wechsler H. (2011) Robust Face Recognition after Plastic Surgery Using Local Region Analysis. In: Kamel M., Campilho A. (eds) Image Analysis and Recognition. ICIAR 2011. Lecture Notes in Computer Science, vol 6754. Springer, Berlin, Heidelberg, Online ISBN: 978-3-642-21596-4, Print ISBN: 978-3-642-21595-7, DOI: https://doi.org/10.1007/978-3-642-21596-4_20

2. Minal Mun and Anil Deorankar(2014), "Implementation of Plastic Surgery Face Recognition Using Multimodal Biometric Features", International Journal of Computer Science and Information Technologies, Vol. 5 (3) , 2014, 3711-3715, ISSN: 0975-9646

3. D. Chawla, M.C. Trivedi (2018), "A Comparative Study on Face Detection Techniques for Security Surveillance" In: Bhatia S., Mishra K., Tiwari S., Singh V. (eds) Advances in Computer and Computational Sciences. Advances in Intelligent Systems and Computing, vol 554. Springer, Singapore, Online ISBN978-981-10-37733, Print ISBN978-981-10-3772-6, DOI: https://doi.org/10.1007/978-981-10-3773-3_52

4. R. Singh, M. Vatsa, H. S. Bhatt, S. Bharadwaj, A. Noore and S. S. Nooreyezdan(2010), "Plastic Surgery: A New Dimension to Face Recognition," in IEEE Transactions on Information Forensics and Security, vol. 5, no. 3, pp. 441-448, Sept. 2010. DOI: 10.1109/TIFS.2010.2054083

5. Dimple Chawla, M.C. Trivedi (2018), "A Review Paper on Face Recognition for Security Surveillance of surgically altered faces", Journal Of Advanced Research In Dynamical and Control Systems, 12-Special Issue Year 2018, pp. 1300-1305, ISSN 1943-023X

6. MariaDe Marsico, MicheleNappi, DanielRiccio, HarryWechsler (2015) "Robust face recognition after plastic surgery using region-based approaches", Pattern Recognition, Volume 48, Issue 4, April 2015, Pages 1261-1276, ISSN: 0031-3203, DOI https://doi.org/10.1016/j.patcog.2014.10.004

7. Jolliffe I.T.(2002), "Principal Component Analysis", Series: Springer Series in Statistics, 2nd ed., Springer, NY, 2002, XXIX, 487 p. 28 illus. ISBN 978-0-38795442-4

8. Hyunjong Cho, Rodney Roberts, Bowon Jung, Okkyung Choi and Seungbin Moon (2014) An Efficient Hybrid Face Recognition Algorithm Using PCA and GABOR Wavelets, International Journal of Advanced Robotic Systems, DOI: 10.5772/58473

9. Vaibhav R. Wagare, Harish K. Bhangale (2015) Plastic Surgical Face Recognition Using Multimode Approach, International Journal of Advanced Research in Electrical, Electronics and Instrumentation Engineering Vol. 4, Issue 7, July 2015, ISSN (Print) : 2320 - 3765ISSN (Online): $2278 \quad-\quad 8875, \quad$ DOI: 10.15662/ijareeie.2015.0407072

10. A. S. O. Ali, V. Sagayan, A. Malik and A. Aziz, "Proposed face recognition system after plastic surgery," in IET Computer Vision, vol. 10, no. 5, pp. 342-348, 8 2016. doi: 10.1049/iet-cvi.2014.0263

M. De Marsico, M. Nappi and D. Riccio, "FARO: FAce Recognition Against Occlusions and Expression Variations," in IEEE Transactions on Systems, Man, and Cybernetics - Part A: Systems and Humans, vol. 40, no. 1, pp. 121-132, Jan. 2010. doi: 
10.1109/TSMCA.2009.2033031

11. Lahasan, B., Lutfi, S.L. \& San-Segundo, R. Artif Intell Rev (2017), "A survey on techniques to handle face recognition challenges: occlusion, single sample per subject and expression", Print ISSN0269-2821, Online ISSN1573-7462, DOI: https://doi.org/10.1007/s10462017-9578-y

12. Pankaj Shivdayal Wasnik, Kiran B. Raja, R. Raghvendra and Christoph Busch (2016) "Eye region based multibiometric fusion to mitigate the effects of body weight variations in face recognition", 19th International Conference on Information Fusion Heidelberg, Germany - July 5-8, 2016

13. Dimple Chawla (2017), "Medical Image Compression using MATLAB", Vivekananda Journal of Research January- June 2017, Vol. 6, Issue 1, 122-135, ISSN 2319-8702(Print) ISSN 2456-7574(Online).

\section{AUTHORS PROFILE}

Dimple Chawla Research Scholar, PAHER University, Udaipur (Rajasthan), INDIA

Email Id: chawladel@gmail.com

Dr. Munesh Chandra TRIVEDI, Dean (Academics), Rajkiya Engineering College, Azamgarh (Uttar Pradesh), INDIA

Email Id: munesh.trivedi@gmail.com 